\title{
Matrix-assisted Laser Desorption Ionization Mass Spectrometry of Maltohexaose and Acetylsalicylic Acid Using Alkali Metal Cation-substituted Zeolites
}

\author{
Junya Suzuki and Tatsuya FuJINo ${ }^{\dagger}$ \\ Department of Chemistry, Graduate School of Science and Engineering, Tokyo Metropolitan University, \\ 1-1 Minami-Osawa, Hachioji, Tokyo 192-0397, Japan
}

\begin{abstract}
Using alkali-metal cation-substituted zeolites and 2,4,6-trihydroxyacetophenone (THAP), which is a typical organic matrix molecule for matrix-assisted laser desorption ionization (MALDI), mass spectrometry has been performed for maltohexaose and acetylsalicylic acid, and the cation-selective ionization of these analytes was achieved. It is found that a complex of cation-substituted zeolite and THAP can be applicable to a compound that is hard to be ionized by a proton adduction in conventional MALDI.
\end{abstract}

(Received June 1, 2012; Accepted July 23, 2012; Published September 10, 2012)

\section{Introduction}

Matrix-assisted laser desorption ionization (MALDI) in combination with time-of-flight (TOF) mass spectrometry (MALDI-MS) is widely used in many research fields, since it enables the observation of analyte ions by their molecular weights. $^{1,2}$ MALDI-MS offers several advantages for the analysis of biological polymers, such as proteins and peptides, but it is not suited to study of low-molecular-weight compounds. In MALDI-MS, an analyte is mixed with a large amount of matrix molecules, and the solid-state mixture is excited typically with UV lasers. The analyte undergoes soft ionization, in which the analyte does not dissociate; however, the matrix molecules that absorb photons from an excitation laser dissociate, which producing many matrix-related peaks in the low-molecular-weight region.

Despite voluminous efforts exerted in previous works, ${ }^{3-7}$ both desorption and ionization mechanisms are still unclear, and a lack of fundamental knowledge has hindered the detection of many kinds of molecules by this method. Even for analytes that can be measured by MALDI-MS, the choice of matrix molecules suited for a particular study is mostly done by trial and error. To solve these problems, a number of attempts have been made by using co-matrices. ${ }^{8-13}$ Matrix-free techniques have been proposed as well. ${ }^{14}$ Recently, we used cyclodextrin ${ }^{15,16}$ and zeolites $^{17}$ as host molecules for typical MALDI matrices, and found that they suppressed the fragmentation of a guest matrix molecule, and increased the peak intensities of protonated analytes.

In this work, we also used zeolites. Zeolites are crystalline aluminosilicates with nano-sized cages, and usually act as solid acid catalysts for many industrial processes. They have high catalytic activity due to a charge imbalance at $\mathrm{Si}-\mathrm{O}-\mathrm{Al}$ bridging

† To whom correspondence should be addressed.

E-mail: fujino@tmu.ac.jp sites, and those sites are compensated by cations. In this study, we exchanged the cations on a zeolite surface with $\mathrm{Na}^{+}, \mathrm{K}^{+}$, and $\mathrm{Cs}^{+}$to apply for analytes that are hard to be ionized by proton adduction. Conventional MALDI as well as our previous method using proton-type zeolite, which gives a proton to an analyte, are not applicable to some compounds such as organic acids and biologically active substances. By using a 2,4,6-trihydroxyacetophenone (THAP) adsorbed on cationsubstituted zeolite (zeolite matrix hereafter), we observed ion peaks of $\mathrm{Na}^{+}, \mathrm{K}^{+}, \mathrm{Cs}^{+}$-adducted analytes. It was also found that the zeolite matrix is applicable to acetylsalicylic acid (ASA), which is hard to be ionized by conventional MALDI.

\section{Experimental}

Mordenite zeolite (JRC-Z-HM20) was supplied by Catalysis Society of Japan (CSJ). Zeolite HM20 was calcined for $6 \mathrm{~h}$ at $773 \mathrm{~K}$ in air before use. Each of sodium nitrite, potassium nitrite, and cesium acetate $(0.3 \mathrm{~mol})$ was dissolved in distilled water $(50 \mathrm{~mL})$ to obtain a $6.0 \mathrm{~mol} \mathrm{~L}^{-1}$ solution. Zeolite HM20 $(300 \mathrm{mg}$ ) was added to the solution, and the mixture was stirred for $1 \mathrm{~h}$ at $353 \mathrm{~K}$. The mixture was filtered at room temperature and the thus-treated zeolite was dispersed again in a sodium nitrite, potassium nitrite, or cesium acetate aqueous solution, and the mixture was stirred for $1 \mathrm{~h}$ at $353 \mathrm{~K}$. We repeated this treatment three times. After filtering, the residue was washed with distilled water $(10 \mathrm{~mL})$ and the mixture was heated for $3 \mathrm{~h}$ at $723 \mathrm{~K}$. Finally, $\mathrm{Na}^{+}$, $\mathrm{K}^{+}$, and $\mathrm{Cs}^{+}$-substituted zeolites (NaM20, KM20, and CsM20) were obtained. THAP, a typical organic MALDI matrix, was recrystallized from methanol before use. THAP (4 mg) and one kind of alkali metal cation-substituted zeolite $(8 \mathrm{mg})$ were mixed in a mortar and pestle. The obtained mixture, zeolite matrix, was named THAP/NaM20, THAP/KM20, or THAP/CsM20, in which THAP molecules were introduced to a non-evacuated zeolite surface where water molecules in the atmosphere were pre-adsorbed. 


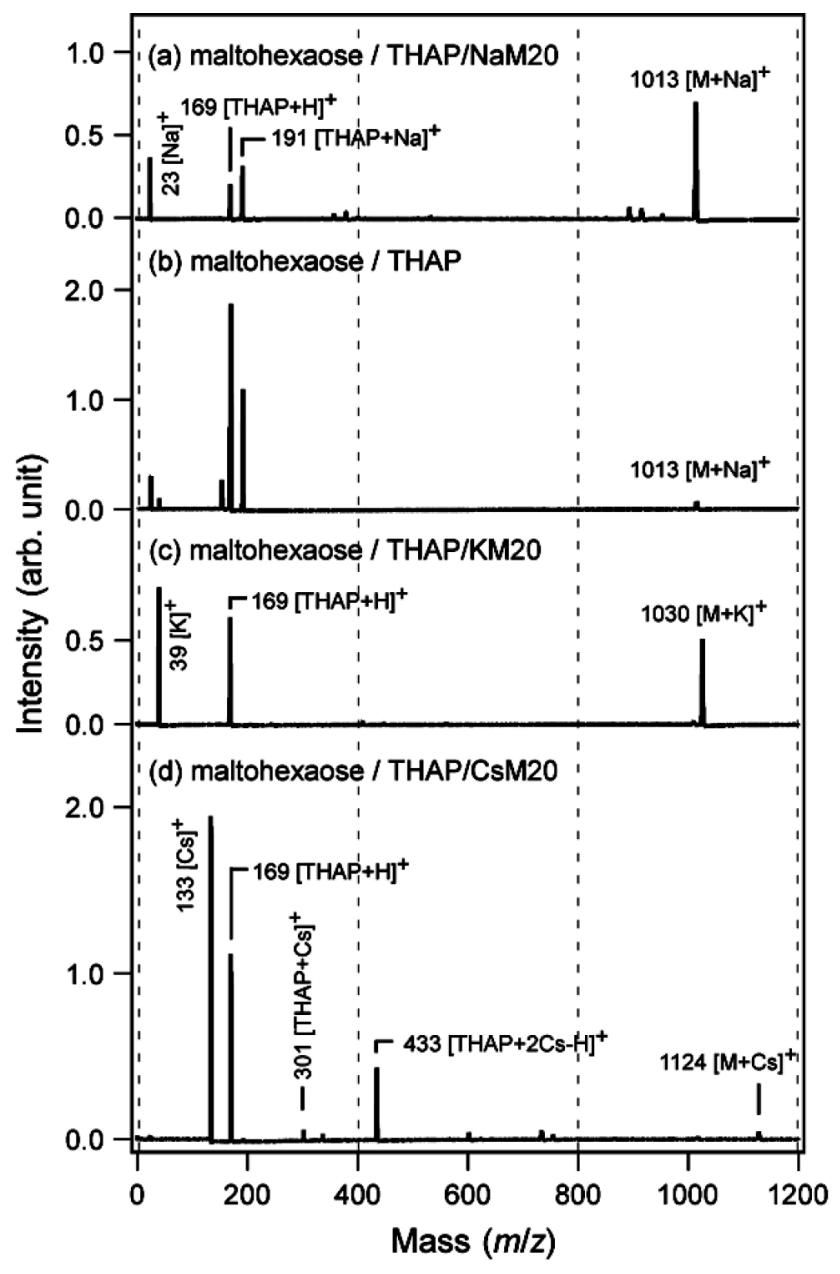

Fig. 1 Mass spectra of maltohexaose (M) measured with (a) THAP/NaM20, (b) THAP only, (c) THAP/KM20, and (d) THAP/CsM20.

The zeolite matrices were suspended in a 1-mL solution of acetonitrile and water $(7: 3, \mathrm{v} / \mathrm{v})$. One microliter each of the zeolite matrix and analyte solution $\left(0.50 \mathrm{mg} \mathrm{mL}^{-1}\right)$ was pipetted onto a stainless plate, left in air for a few minutes to evaporate the solvent, and analyzed by a laboratory-built MALDI mass spectrometer. A Nd:YAG laser (Spectra Physics, $266 \mathrm{~nm}$, $10 \mathrm{~Hz}, 2 \mathrm{~ns}$ ) was used as the excitation light, and the typical pulse energy was $5 \mu \mathrm{J}$. Produced ions from the sample were accelerated with an electric field of $2.3-4.0 \mathrm{kV}$, and spectra were acquired with a linear TOF tube and an MCP detector.

\section{Results and Discussion}

Figure 1(a) shows the mass spectrum of maltohexaose measured with a THAP/NaM20. The peaks of protonated and $\mathrm{Na}^{+}$-adducted THAP were observed. No fragmentation peaks of THAP were found, although a strong peak of sodium ion is noted at $\mathrm{m} / \mathrm{z}=$ 23. In regard to the analyte, the peak of $\mathrm{Na}^{+}$-adducted maltohexaose (M) is clearly observed at $\mathrm{m} / \mathrm{z}=1013$, indicating ionization by alkali metal cation adduction on the zeolite surface. The mass spectrum of maltohexaose measured only with THAP (conventional MALDI) is shown in Fig. 1(b) for a comparison. It is understood that the peak intensity of analyte-related ion is very weak in conventional MALDI. In the case of the zeolite matrix, however, the peak intensity of $[\mathrm{M}+\mathrm{Na}]^{+}$is quite strong, i.e., almost two-times the matrix-related peak intensities shown in Fig. 1(a). Suppression of the fragmentation of matrix molecules and an enhancement of the analyte peak intensity are advantages of our zeolite matrix, as we reported in our previous paper concerning THAP/HM20. ${ }^{17}$ Mass measurements of maltohexaose were also carried out with THAP/KM20 (Fig. 1(c)) and THAP/CsM20 (Fig. 1(d)). With regard to the analyte, the peak of $\mathrm{K}^{+}$-adducted maltohexaose was observed at $\mathrm{m} / \mathrm{z}=1030$, but it is less prominent than that of $[\mathrm{M}+\mathrm{Na}]^{+}$in Fig. 1(a). In the case of THAP/CsM20 in Fig. 1(d), a very weak peak of $[\mathrm{M}+\mathrm{Cs}]^{+}$can be seen. Therefore, ionization of the analyte is achieved according to the type of cation on the zeolite surface, also the ionized analyte peak intensity becomes weak as the atomic mass of the cation is increased.

Peaks of protonated THAP are observed in the three spectra. However, those of the protonated analyte are not observed. This means that cation adduction from $[\mathrm{THAP}+\mathrm{Na}, \mathrm{K}, \mathrm{Cs}]^{+}$to analyte overrode $\mathrm{H}^{+}$adduction from $[\mathrm{THAP}+\mathrm{H}]^{+}$to the analyte. It can be understood that the binding energy between THAP and an alkali metal cation becomes low as the electrostatic force becomes low along with an increase of the ionic radius. Therefore, it is considered that compared to proton, alkali metal cations $\left(\mathrm{Na}^{+}, \mathrm{K}^{+}, \mathrm{Cs}^{+}\right)$would easily detach from $[\mathrm{THAP}+\mathrm{Na}, \mathrm{K}, \mathrm{Cs}]^{+}$to produce $[\text {analyte }+\mathrm{Na}, \mathrm{K}, \mathrm{Cs}]^{+}$. In addition, it is also found that the peak intensities of cation-adducted matrix $\left([\mathrm{THAP}+\mathrm{K}, \mathrm{Cs}]^{+}\right)$became weak or disappeared when zeolites with $\mathrm{K}^{+}$and $\mathrm{Cs}^{+}$were used. In the MALDI process, it is considered that matrix molecules desorb with excess energy, since the matrix absorbs photons of the excitation laser, whereas the analyte undergoes soft ionization and desorption with a low excess energy. Therefore, it is assumed that ions of $[\mathrm{THAP}+\mathrm{K}, \mathrm{Cs}]^{+}$would be detached by excess energy during desorption process, and this can be confirmed by the fact that the peak intensity of $\mathrm{K}^{+}$and $\mathrm{Cs}^{+}$at $\mathrm{m} / \mathrm{z}=39$ and 133 became larger with increasing of atomic number.

In previous research of the zeolite matrix with protonated zeolite (THAP/HM20), we concluded that both the acidic zeolite surface and adsorbed water molecules enhanced proton adduction to the analyte molecules by comparing the peak intensities in the mass spectra measured with zeolite matrices prepared with and without evacuation procedures. ${ }^{17}$ By analogy, we consider that cation adduction from a zeolite surface to an analyte via a matrix molecule is also enhanced. In this research, since we used alkali metal cation-substituted zeolite, it is assumed that the peak of protonated THAP comes from conventional MALDI-MS, in which the acidity of the zeolite surface is not used. Therefore, analyte ionization by a cation originating from the zeolite is governed, whereas the peak of a protonated analyte that may originate from the usual MALDI is not observed.

Figure 2(a) shows the mass spectrum of maltohexaose measured with only NaM20 (without THAP). Only the peak of the sodium ion appearing at $\mathrm{m} / \mathrm{z}=23$ is observed. Such zeolites as NaM20 do not show any absorption in the UV-vis region. Therefore, zeolite does not work as a matrix by itself, and a matrix molecule, such as THAP, is necessary to ionize the analyte. Cation or proton relay to the analyte takes place only from the matrix molecule and the direct cation transfer from the zeolite to the analyte can be ruled out. When anthracene molecules having an absorption coefficient at $266 \mathrm{~nm}$ are used instead of THAP, the peak of analyte (maltohexaose) is not observed, although the peak of an anthracene cation, [anthracene] ${ }^{+}$, is observed at $\mathrm{m} / z=178$ (Fig. 2(b)). Therefore, the matrix molecule should have not only an absorption band in 


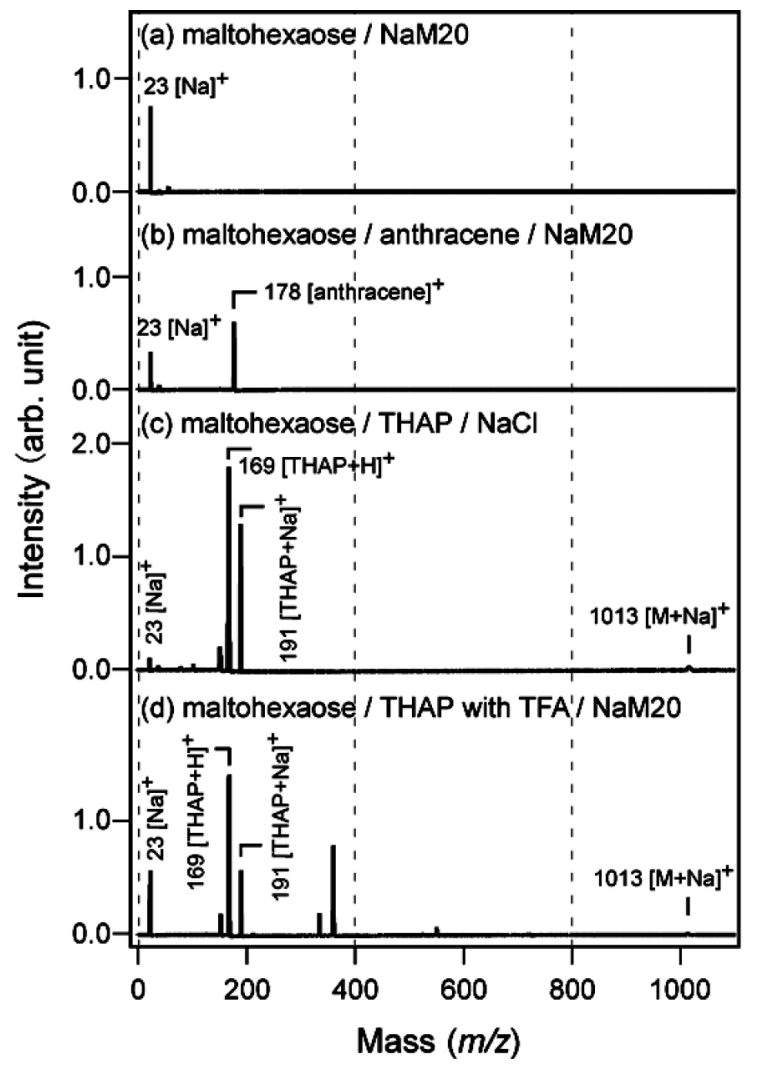

Fig. 2 Mass spectra of maltohexaose measured with (a) NaM20 only and (b) anthracene/NaM20, in which anthracene was used as the matrix instead of THAP. (c) Mass spectrum of maltohexaose measured with THAP and $\mathrm{NaCl}$. $\mathrm{NaCl}$ was used instead of cation-substituted zeolite. (d) Mass spectrum of maltohexaose measured with THAP/NaM20 including TFA.

the wavelength region of the excitation laser, but also cation adduction ability. Figure 2(c) shows the mass spectrum of maltohexaose with sodium chloride $\left(4 \mathrm{mg} \mathrm{mL}^{-1}\right)$, which is intended to serve as the cation source instead of zeolite. In this example, the peak of protonated THAP is observed, but that of the protonated analyte is not. This means that sodium chloride does not work as a cation source, and causes a suppression of the peak of the protonated analyte. Analyte ion peak suppression is also observed when trifluoroacetic acid (TFA) is used. TFA is widely used as a cationization agent in conventional MALDI-MS. In mass measurements with our zeolite matrix, however, the peak of the $\mathrm{Na}^{+}$-adducted analyte is suppressed, as shown in Fig. 2(d). Because of electron-withdrawing effect of fluorines, a proton in TFA can easily be detached. With the presence of protons from TFA, it is considered that alkali metal cations on acidic zeolite cannot be easily detached, and zeolite cannot be used as an efficient cation source for the ionization of THAP and the analyte. Therefore, we cannot use TFA in mass measurements with the zeolite matrix.

In addition to the two functions of the zeolite matrix, namely, suppression of the matrix molecule fragmentation and enhancement of the analyte peak intensity, we introduce another function: a cation-substituted zeolite matrix can be applied to compounds that are difficult to ionize and observe by conventional MALDI-MS. Figure 3(a) shows the mass spectrum of ASA measured with only THAP (conventional MALDI-MS). ASA is one of the representative molecules that cannot be measured by conventional MALDI-MS. Although the peaks of

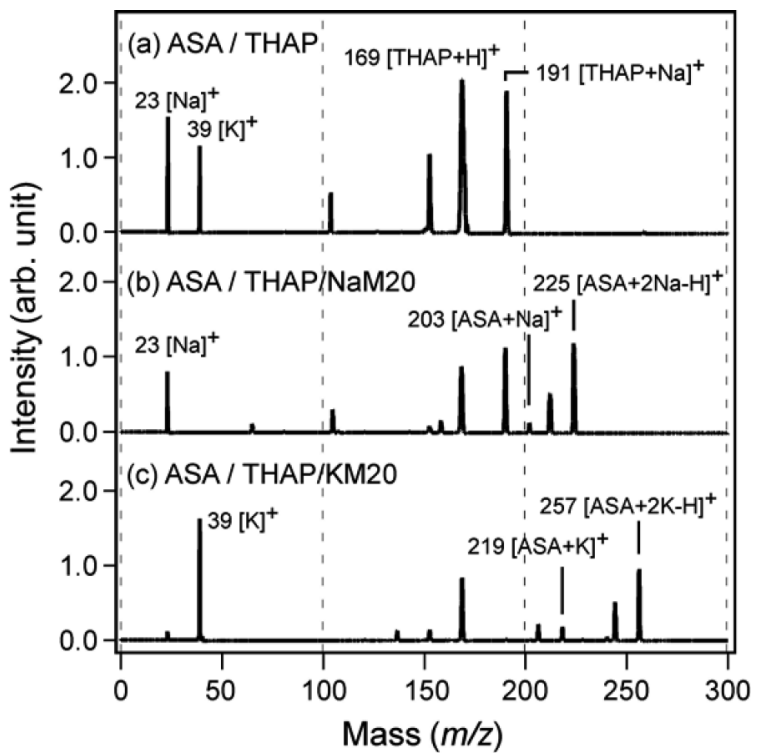

Fig. 3 Mass spectra of acetylsalicylic acid (ASA) measured with (a) THAP (conventional), (b) THAP/NaM20, and (c) THAP/KM20.

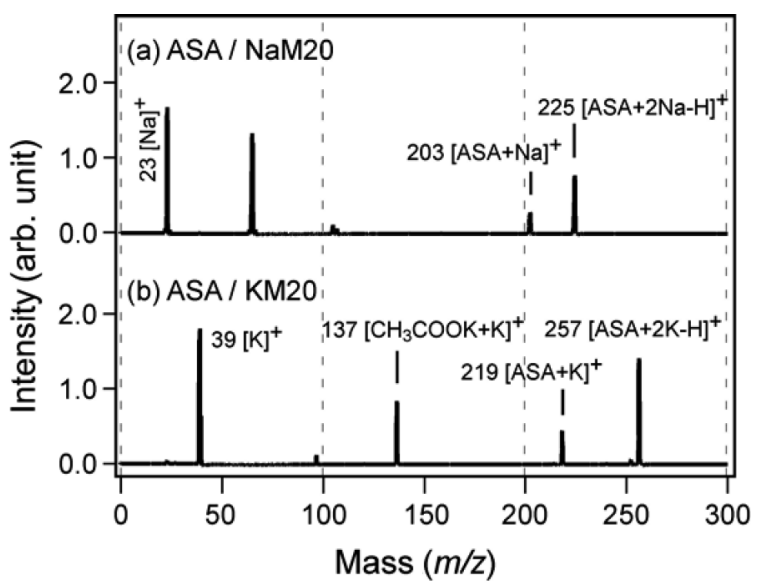

Fig. 4 Mass spectra of acetylsalicylic acid measured with (a) NaM20 only and (b) KM20 only.

protonated and $\mathrm{Na}^{+}$-adducted THAP are observed, the peak of the analyte cannot be detected (exact mass of ASA is 180.16). By using zeolite matrices, however, ASA can be ionized without decomposition through alkali metal ion adduction. Figure 3(b) shows the mass spectrum of ASA measured with THAP/NaM20. In addition to the matrix-related peaks, the peaks of $\mathrm{Na}^{+}$-adducted ASA are clearly observed at $m / z=203$ and 225. The peaks of ASA can be observed as well by using THAP/KM20 (Fig. 3(c)). The peaks of $\mathrm{K}^{+}$-adducted ASA are observed at $\mathrm{m} / \mathrm{z}=219$ and 257. Therefore, the zeolite matrix is applicable to acidic compounds, such as ASA, to which protons hardly adduct. In Figs. 3(b) and 3(c), peaks such as $[\mathrm{ASA}+2 \mathrm{Na}-\mathrm{H}]^{+}$or $[\mathrm{ASA}+2 \mathrm{~K}-\mathrm{H}]^{+}$are observed strongly rather than $[\mathrm{ASA}+\mathrm{Na}]^{+}$or $[\mathrm{ASA}+\mathrm{K}]^{+}$. It can be assumed that there are two adduction sites for alkali metal cation with almost equal binding energy.

Figure 4(a) shows the mass spectrum of ASA measured with only NaM20. In a similar manner to Fig. 3(b), the peaks of $\mathrm{Na}^{+}$-adducted ASA are observed at $\mathrm{m} / \mathrm{z}=203$ and 225, which means that the analyte ASA can be used as a matrix molecule at 
the same time. ASA is one of the organic acid compounds to which protons hardly adduct. However, ASA can be used as a matrix molecule to assist in cation adduction. By using KM20, ASA can be observed as $\mathrm{K}^{+}$-adducted species, as shown in Fig. 4(b). Since the zeolite surface acts as a super strong acid, we are convinced that the zeolite can be used with compounds that do not prefer proton, thus further improving the applicability of MALDI-MS.

\section{Conclusions}

An efficient matrix for MALDI-MS was developed. A complex of a typical organic matrix (THAP) and cation-substituted zeolite, called zeolite matrix, was used for mass spectrometric measurements of maltohexaose, and cation-selective ionization of the analyte was achieved. The roles of THAP and zeolite were confirmed through several experiments: (1) matrix molecules, such as THAP, should have an absorption coefficient at the excitation wavelength and cation adduction ability at the same time; (2) zeolite is an effective cation source compared to salt; and (3) TFA suppresses the production of cation-adducted analyte. We found that the developed zeolite matrix was applicable to ASA, the ions of which are difficult to observe by conventional MALDI-MS.

\section{Acknowledgements}

T. F. acknowledges a Grant-in-Aid for Scientific Research on Priority Area (No. 477) from MEXT and a Grant-in-Aid for Scientific Research (C) (No. 24550030) from JSPS.

\section{References}

1. K. Tanaka, H. Waki, Y. Ido, S. Akita, Y. Yoshida, and T.
Yoshida, Rapid Commun. Mass Spectrom., 1988, 2, 151.

2. F. Hillenkamp, M. Karas, R. C. Beavis, and B. T. Chait, Anal. Chem., 1991, 63, 1193A.

3. R. Kruger, A. Pfenninger, I. Fournier, M. Gluckmann, and M. Karas, Anal. Chem., 2001, 73, 5812.

4. M. Karas and R. Kruger, Chem. Rev., 2003, 103, 427.

5. M. Karas, M. Gluckmann, and J. Schafer, J. Mass Spectrom., 2000, 35,1 .

6. H. Ehring, M. Karas, and F. Hillenkamp, Org. Mass Spectrom., 1992, 27, 427.

7. W. C. Chang, L. C. L. Huang, Y.-S. Wang, W.-P. Peng, H. C. Chang, N. Y. Hsu, W. B. Yang, and C. H. Chen, Anal. Chim. Acta, 2007, 582, 1.

8. X. Yang, H. Wu, T. Kobayashi, R. J. Solaro, and R. B. v. Breemen, Anal. Chem., 2004, 76, 1532.

9. T. Nishikaze and M. Takayama, Rapid Commun. Mass Spectrom., 2007, 21, 3345.

10. C. Koester, J. A. Castoro, and C. L. Wilkins, J. Am. Chem. Soc., 1992, 114, 7572.

11. T. Kobayashi, H. Kawai, T. Suzuki, T. Kawanishi, and T. Hayakawa, Rapid Commun. Mass Spectrom., 2004, 18, 1156.

12. S. Kjellstroem and O. N. Jensen, Anal. Chem., 2004, 76, 5109 .

13. J. Asara and J. Allison, J. Am. Soc. Mass Spectrom., 1999, 10,35 .

14. R. Arakawa, Y. Shimomae, H. Morikawa, K. Ohara, and S. Okuno, J. Mass Spectrom., 2004, 39, 961.

15. S. Yamaguchi, T. Fujita, T. Fujino, and T. Korenaga, Anal. Sci., 2008, 24, 1497.

16. T. Fujita, T. Fujino, K. Hirabayashi, and T. Korenaga, Anal. Sci., 2010, 26, 743.

17. Y. Komori, H. Shima, T. Fujino, J. N. Kondo, K. Hashimoto, and T. Korenaga, J. Phys. Chem. C, 2010, 114, 1593. 